\title{
$\mathbf{1}_{\text {st }}$ Reading
}

July 1, 2007 22:44 WSPC/115-IJPRAI SPI-J068 00569

\author{
1 International Journal of Pattern Recognition \\ and Artificial Intelligence \\ 3 Vol. 21, No. 5 (2007) 1-22 \\ (C) World Scientific Publishing Company
}

\section{GAZE TRACKING SYSTEM MODEL BASED ON PHYSICAL PARAMETERS}

In the past years, research in eye tracking development and applications has attracted much attention and the possibility of interacting with a computer employing just gaze information is becoming more and more feasible. Efforts in eye tracking cover a broad spectrum of fields, system mathematical modeling being an important aspect in this research. Expressions relating to several elements and variables of the gaze tracker would lead to establish geometric relations and to find out symmetrical behaviors of the human eye when looking at a screen. To this end a deep knowledge of projective geometry as well as eye physiology and kinematics are basic. This paper presents a model for a bright-pupil technique tracker fully based on realistic parameters describing the system elements. The system so modeled is superior to that obtained with generic expressions based on linear or quadratic expressions. Moreover, model symmetry knowledge leads to more effective and simpler calibration strategies, resulting in just two calibration points needed to fit the optical axis and only three points to adjust the visual axis. Reducing considerably the time spent by other systems employing more calibration points renders a more attractive model.

Keywords: Eye tracking; modeling; calibration; bright-pupil technique; optical axis; visual axis.

\section{Introduction}

31 Several years ago, the initial to first steps to track human gaze were launched. ${ }^{23,24}$ Eye properties, like corneo-retinal potential difference, were employed by Mowrer et al. ${ }^{13}$ and other novel techniques were introduced by Cornsweet and Crane ${ }^{4}$ or by Merchant et al. ${ }^{11}$ May be at that time the possibility of controlling a computer using just the gaze was quite unimaginable but, due to the increasing capabilities of eye tracking systems achieved since then, such ideas are rapidly becoming more and more realistic as in the work of Jacob. ${ }^{10}$

The purpose of a gaze tracking system is mainly to determine the gaze direction of the person using the system. These techniques are being improved to achieve more robust systems that are reliable and provide the gaze position with the highest 


\section{$\mathbf{1}_{\text {st }}$ Reading}

precision, videoculography (VOG) techniques are some of the most popular. It is well known that VOG consists basically in employing some illumination method and image acquisition to determine, through the captured images, the point the subject is looking at. One of its remarkable features is the possibility of building nonintrusive systems that avoid the use of helmets or special glasses. The number of cameras or the type of illumination employed allow different system possibilities; from the simplest configuration that consists on one unique camera and illumination source, ${ }^{22}$ to more elaborated systems making use of stereo vision techniques or exploring different illumination capabilities such as polarized light. ${ }^{19}$

An interesting aspect of eye tracking research is the modeling of the eye-gaze tracking system. A lot of work has been devoted to design a mathematical model able to describe the relation among the elements that take part in a tracking session, such as subject's eye, screen, camera and illumination. Dynamics of eyeball and more generally its physiology are a widely studied topic. Although the schematic description of the eyeball is well known by the eye tracking community, this knowledge has hardly been applied to gaze tracker design. In other words, among the different models of the eyeball presented during years (a good review can be found in the book by Rabbetts ${ }^{17}$ ) it is still not clear which of them keeps best the compromise between simplicity and effectiveness when it has to be employed in an eye-gaze tracking system. Models used to describe gaze direction do not reflect the behavior of the eye in the most realistic possible way. In this field Beymer and Flickner ${ }^{1}$ or Shih and $\mathrm{Liu}^{18}$ present different answers for the same matter, meanwhile $\mathrm{Ohno}^{15}$ and $\mathrm{Ott}^{16}$ would be remarkable examples. However, gaze tracking mathematical modeling is maybe one of the least explored fields of eye-movement research. Generic mathematical expressions, like linear or quadratic forms based on undefined coefficients, are normally employed to relate the point $\left(p_{x v}, p_{y v}\right)$ to which the subject is focused with specific features extracted from the image. ${ }^{11}$ Long recognized concepts like the difference between the optical and visual axes of the eye and more frequently the "false torsion" concept, that will be later more extensively explained, are usually obviated. Deeply studied Listing's and Donder's ${ }^{17}$ laws describe the eyeball movements with high exactness, for example in Refs. 6, 7 and 14. It seems reasonable to expect that a model able to reflect not only the behavior of the eye but also the relative location of the elements composing the system would exhibit a more robust performance.

Such is the objective of this work, i.e. to design a mathematical model, totally based on parameters describing the elements that compose an eye tracking system, involving knowledge about eyeball physiology and dynamics. The aim is to review mathematically the minimum number of parameters of the eye that are necessary to build up a feasible model using the minimum number of image features. The model will start with the most simplistic schematic description of the eye and it will add parameters to include aspects of the eye physiology and kinematics that can lead to a valid tracking system. The work performs both optical and visual axes description and includes as novelty the well known "false torsion" modeling. 


\section{$\mathbf{1}_{\text {st }}$ Reading}

July 1, $2007 \quad$ 22:44 WSPC/115-IJPRAI $\quad$ SPI-J068 00569

Eye Tracking System Model Based on Physical Parameters

1

3

On another hand, calibration is one of the most tiresome processes that precede any tracking session. The subject is asked to look at a set of markers on the screen, what allows adjusting model parameters in order to fit the system to the user and tracker set up. Deeper knowledge about the model would allow a faster and more efficient calibration. A direct outcome of our task has been to design better calibration strategies; just two points are enough to calibrate the model for the optical axis and only three points are needed to adjust the proposed system to track the visual axis. The performance achieved with three points is comparable to that accomplished by other generic models that employ more calibration points for fitting purposes. Basically the pursued model has to be fully based on parameters describing elements of the system, it has to be easy to implement and provide the most simple calibration.

In the next section the system to be analyzed is presented. Eye modeling is one of the most complex matters in the searching for the final objective, so a special section is dedicated to it. Section 4 starts with the modeling of this system, defining and naming the variables that describe the several elements involved in a tracking session. Once the model is completed in Sec. 5, Sec. 6 is fully devoted to find and justify the best and most efficient calibration strategies. Results are experimentally confirmed in Sec. 7. Lastly a list of conclusions and future work guidelines are proposed.

\section{The Eye Tracking System}

Among the different eye tracking methods employing VOG, the simplest configuration of the bright pupil-technique has been selected. It is well known how this method performs the tracking of the subject's gaze. ${ }^{24}$ The arrangement considered for this work (sketched in Fig. 1) consists basically in employing an illumination source coaxially disposed with the camera, lighting up the eye. A camera with a proper sensitivity to the illumination wavelength captures the resulting images. Infrared light sources are widely employed due to their lack of effects on the user.

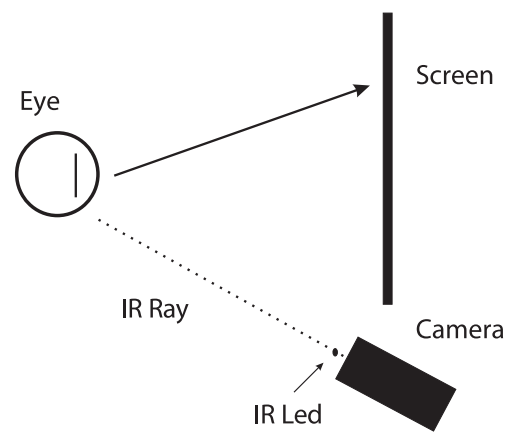

Fig. 1. Elements of the eye tracking system using bright pupil technique. 


\section{$\mathbf{1}_{\text {st }}$ Reading}

This illumination technique produces two interesting features in the image. First, all the light reaching the subject's retina is reflected and captured by the camera, what makes the pupil to appear brighter in the image. Second, a point with higher intensity produced by the reflection of the illumination ray on the subject's cornea can be found.

This technique has been widely employed by different systems and several improvements of the method have been carried out. ${ }^{5,9,12}$ The bright pupil technique employs two parameters extracted from the image: the reflection of the illumination source on the eyeball that produces the brightest pixels in the image and is called glint or corneal reflection, $\left(r_{x}, r_{y}\right)$ and the center of the pupil in the image $\left(m_{x}, m_{y}\right)$.

The difference between both points in the image is called "glint-pupil vector" or "corneal reflection-pupil center vector". ${ }^{24}$

$$
\left(v d_{x}, v d_{y}\right)=\left(r_{x}, r_{y}\right)-\left(m_{x}, m_{y}\right)
$$

This vector represents the working variable of the tracking model. Basically the relation we are looking for can be simply described as shown in Fig. 2. The objective of the work is to express $\left(p_{x v}, p_{y v}\right)$, i.e. the point being observed, as a function of the different parameters describing the elements of the system together with $\left(v d_{x}, v d_{y}\right)$.

\section{The Eyeball}

Human eye can be considered as the most complex element of the gaze tracking system. It is not the aim of this work to make an exhaustive and accurate description of the eyeball physiology, but strict enough to achieve a feasible model for the human gaze modeling. Eyeball dynamics have been widely studied by Carpenter ${ }^{3}$ and Yarbus ${ }^{23}$ among others (see Ref. 17).

The eye consists of a set of refracting surfaces that are normally considered as spherical. The optical axis can be approximated as the symmetry axis of the eye, where the centers of curvature of these different surfaces are located. ${ }^{17}$

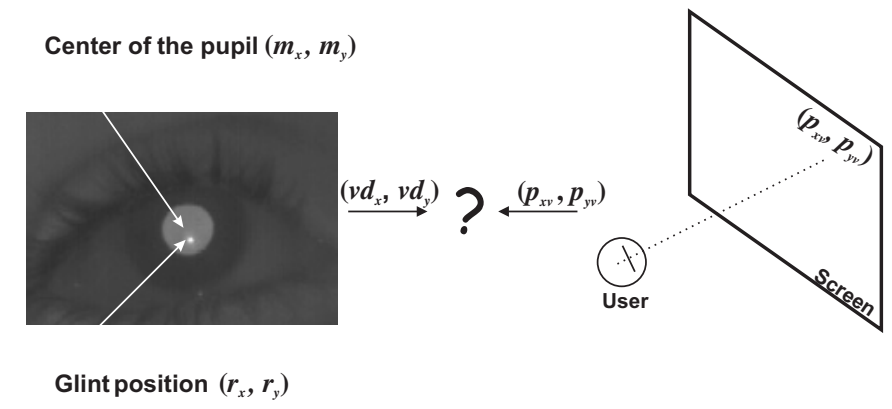

Fig. 2. Model definition, i.e. the mathematical connection between $\left(v d_{x}, v d_{y}\right)$ and gazed point $\left(p_{x v}, p_{y v}\right)$ is pursued. 


\section{$\mathbf{1}_{\text {st Reading }}$}

July 1, $2007 \quad 22: 44 \quad$ WSPC/115-IJPRAI $\quad$ SPI-J068 00569

Eye Tracking System Model Based on Physical Parameters

When looking at a point, the eye is oriented in such a way that the observed object projects itself on the fovea (a small area of the retina with a high density of cones that are responsible of high visual detail discrimination) that is slightly displaced from the eyeball back pole. The line joining the fovea with the object we are looking at can be approximated as the visual axis, that is defined as the line joining the fovea with the nodal point (close to the corneal sphere center). Therefore, gaze modeling is clearly related to visual axis modeling. Due to the offset of the fovea from the eye back pole there is an horizontal angle of $5^{\circ} \pm 2^{\circ}$ between both axes. A lower angle $\left(2^{\circ}-3^{\circ}\right)$ can be specified vertically too, although there is a considerable personal variation. ${ }^{3}$ The difference between optical and visual axes is basic in the design of a tracking system. Figure 3 (left) shows a basic scheme of the left eye top view, where both optical and visual axes are represented.

The requirement of distinguishing between optical and visual axes is accepted by most eye tracking system modelers. ${ }^{1,15}$ However the kinematics of the eyeball rotations are not so strictly considered by designers, although knowledge about eyeball rotation and orientation is important in the design of the model. The relative orientation of visual and optical axes varies as function of different eye movements that have been broadly discussed and described by Listing's and Donder's laws.

As Ferman ${ }^{6}$ explains, different topics have lead to discussion, i.e. the number of degrees of freedom required to express the movement and rotation of the eyeball, the most convenient coordinate system and the "false torsion" among others. The introduction of the "false torsion" concept is necessary to achieve a concurrence with the eye kinematics theory that is briefly described by Fry. ${ }^{7}$ As it is shown in Fig. 3 (right) once the vertical $\theta_{v}$ and horizontal $\varphi_{v}$ rotations are accomplished by the visual axis from its primary position (looking straight ahead, the head being erect) to reach a tertiary one ${ }^{17}$ (vertical plus horizontal rotation) an additional rotation is required around the visual axis to locate the eyeball accordingly with Listing's

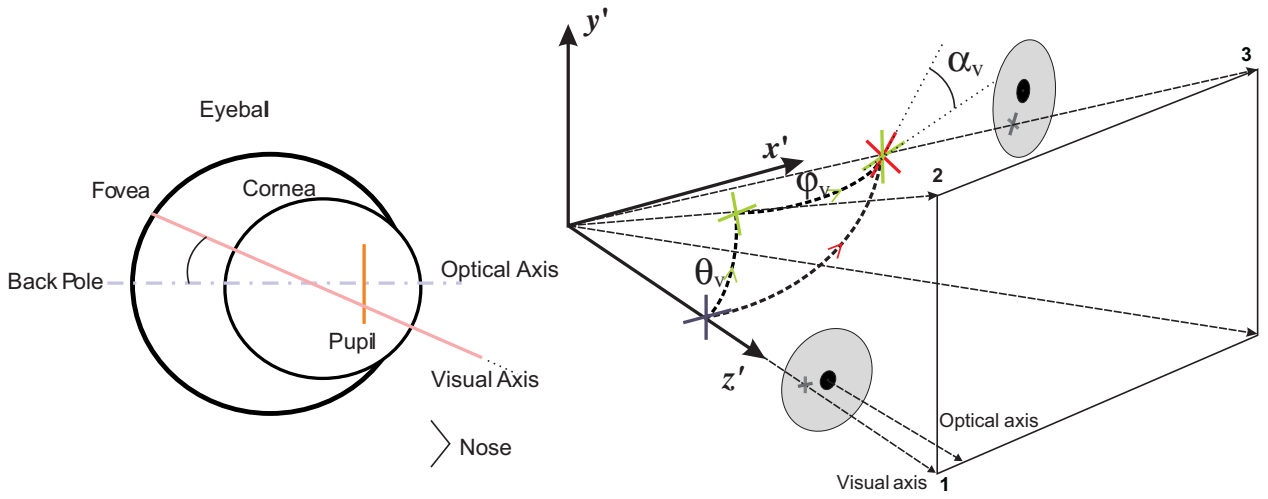

Fig. 3. (Left) Optical axis and visual axis (top view of left eye). (Right) eye rotation from its primary position to a tertiary one modeled in one step (Listing's Law) and by means of consecutive vertical and horizontal rotations. 


\section{$\mathbf{1}_{\text {st }}$ Reading}

July 1, $2007 \quad$ 22:44 WSPC/115-IJPRAI $\quad$ SPI-J068 00569

6

A. Villanueva, R. Cabeza \&S S. Porta

1 Law that describes the 3D orientation of the eyeball for alternative positions. This supplementary rotation depends on the previously rotated angles and is called "false

3 torsion". The eyeball in its primary position (looking at point 1) presents an orientation represented by the cross. As Listing's Law states, when rotating the eye from position (line) 1 to 3 the eye acts in one step, i.e. one rotation movement indicated by the curve connecting $1-3$. If we model the eye movement by means of consecutive vertical $\theta_{v}(1-2)$ and horizontal $\varphi_{v}(2-3)$ rotations, which is more easily handled, the orientation achieved by the cross does not match the correct one, as shown in the figure. An additional rotation $\alpha_{v}$ around the visual axis is necessary to agree with the final orientation, i.e. "false torsion". Employing the expression by $\mathrm{Fry}^{7}$ the torsion around the visual axis can be expressed as,

$$
\tan \left(\frac{\alpha_{v}}{2}\right)=\tan \left(\frac{\theta_{v}}{2}\right) \tan \left(\frac{\varphi_{v}}{2}\right)
$$

Considering anticlockwise rotation positive we have, as shown by Fry, ${ }^{7}$

$$
\sin \alpha_{v}=\frac{-\sin \theta_{v} \sin \varphi_{v}}{1+\cos \theta_{v} \cos \varphi_{v}}
$$

The section that concludes has tried to justify and clarify in a plain form some of the modifications to be accomplished in the final model, i.e. the distinction between eye optical and visual axes and the requirement of introducing the false torsion. A compromise has to be found between competence and simplicity. Readers are encouraged to consult the proposed readings for detailed information about the discussed topics.

\section{System Modeling}

Once the system is presented the mathematical description of its elements has to be accomplished. Basically, the eyeball is located in a $3 \mathrm{D}$ space with a specific orientation, looking to a certain point $\left(p_{x v}, p_{y v}\right)$ on the screen. Likewise, screen, camera and illumination have to be described in some way as well. In the following paragraphs, a set of variables and reference systems is presented, that are employed to describe the elements implied in the selected eye tracker and will participate in the designed final model. A more detailed description can be found in Ref. 22.

A main reference system $\left(x_{2}, y_{2}, z_{2}\right)$ is defined with the origin at the camera projection center. The camera optical axis coincides with $z_{2}$. The remaining elements in the system (screen, illumination and eyeball) take this point as reference.

The eyeball in a first approach is considered as a spherical surface. Its center is referenced to the camera projection center with a set of coordinates $\left(a_{1}\right.$, $\left.a_{2}, a_{3}\right)$ and the screen origin, considered as the top left corner, has coordinates $\left(p o_{x 2}, p o_{y 2}, p o_{z 2}\right)$. As we will see later, both the eye and the screen could rotate with regard to this reference system. The illumination source has also to be referred to this point, its coordinates being named $\left(l_{x 2}, l_{y 2}, l_{z 2}\right)$. Anyway, considering the 


\section{$\mathbf{1}_{\text {st Reading }}$}

July 1, $2007 \quad$ 22:44 WSPC/115-IJPRAI SPI-J068 00569

Eye Tracking System Model Based on Physical Parameters

1 coaxial arrangement of this element, we already know that $l_{x 2}=l_{y 2}=0$ since the lighting will be located in the optical axis of the camera, i.e. $z_{2}$-axis.

3

5

7

9

11

15

17

19

On the other hand, a second reference system $\left(x_{1}, y_{1}, z_{1}\right)$ is placed at the eyeball center, where the pupil is referred. This system rotates together with the eye and its $z_{1}$-axis matches the eye optical axis. The angles $\left(\theta_{o}, \varphi_{o}\right)$ specify the orientation of the eye optical axis with regard to the fixed camera axes. $r$ and $t$ are considered the radius of the pupil and the distance between the eyeball center and the pupil center, respectively. The torsion angle of the eye $\alpha_{o}$ will be later introduced together with eyeball dynamics.

The screen has its own reference system, this being a two-dimensional system, and in the most general arrangement the screen presents three-rotation possibilities with respect to the camera, that will be described by the parameters $\left(\varphi_{p}, \theta_{p}, \alpha_{p}\right)$.

The pupil is projected on the camera image plane. A perspective projection model $^{20}$ is considered for the camera, with the following camera intrinsic parameters, $f$ focal distance, $(u, v)$ image plane center and aspect ratio $\alpha$.

The aim of Fig. 4 is to describe the whole arrangement at one sight showing together each element of the eye tracking system and all the reference systems described in the preceding paragraphs. For simplicity, eye visual axis has been omitted in the figure. Later both axes will be distinguished in the model together with the interesting coordinates on the screen, i.e. $\left(p_{x o}, p_{y o}\right)$ point of the screen

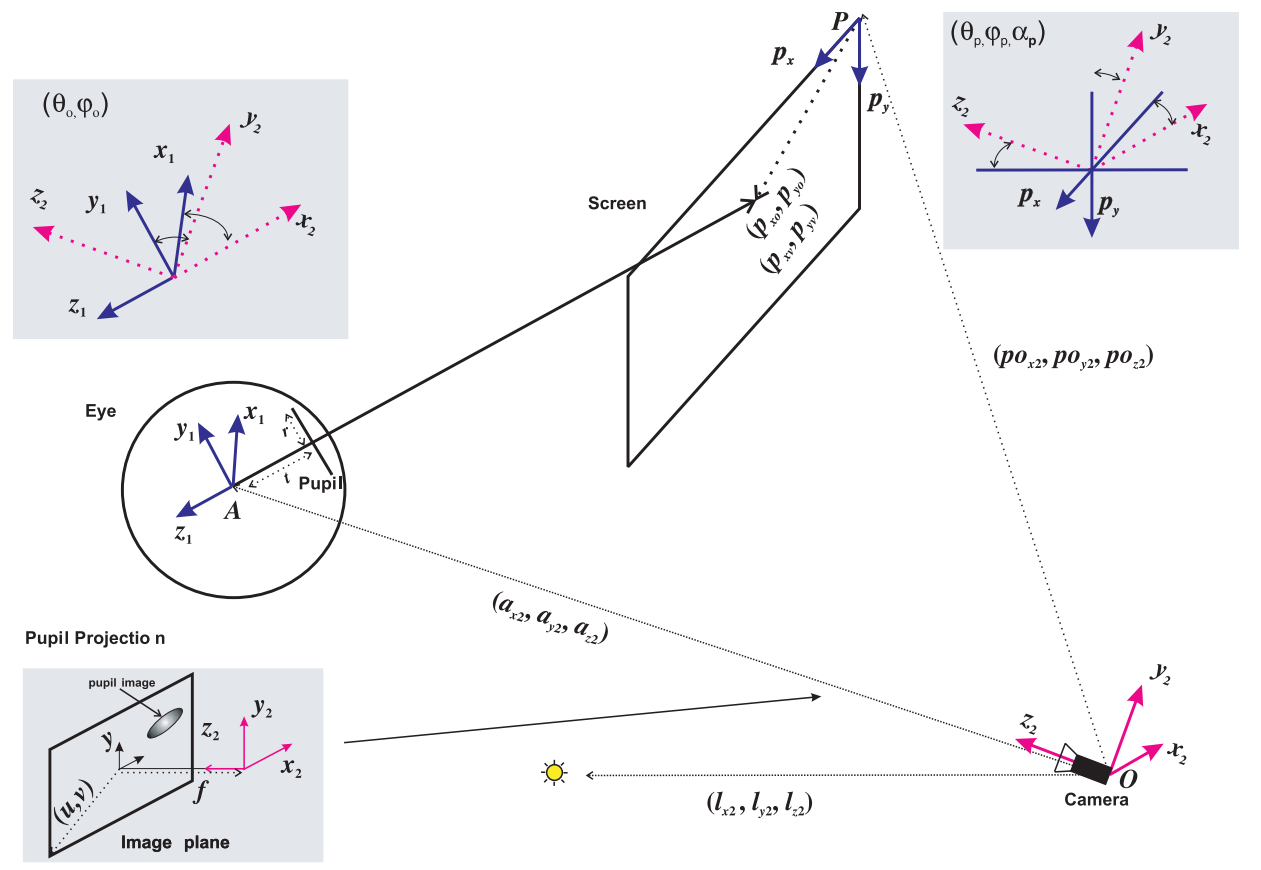

Fig. 4. Eye tracking system geometric description. 


\section{$\mathbf{1}_{\text {st Reading }}$}

July 1, $2007 \quad$ 22:44 WSPC/115-IJPRAI SPI-J068 00569

8

A. Villanueva, R. Cabeza \&S S. Porta

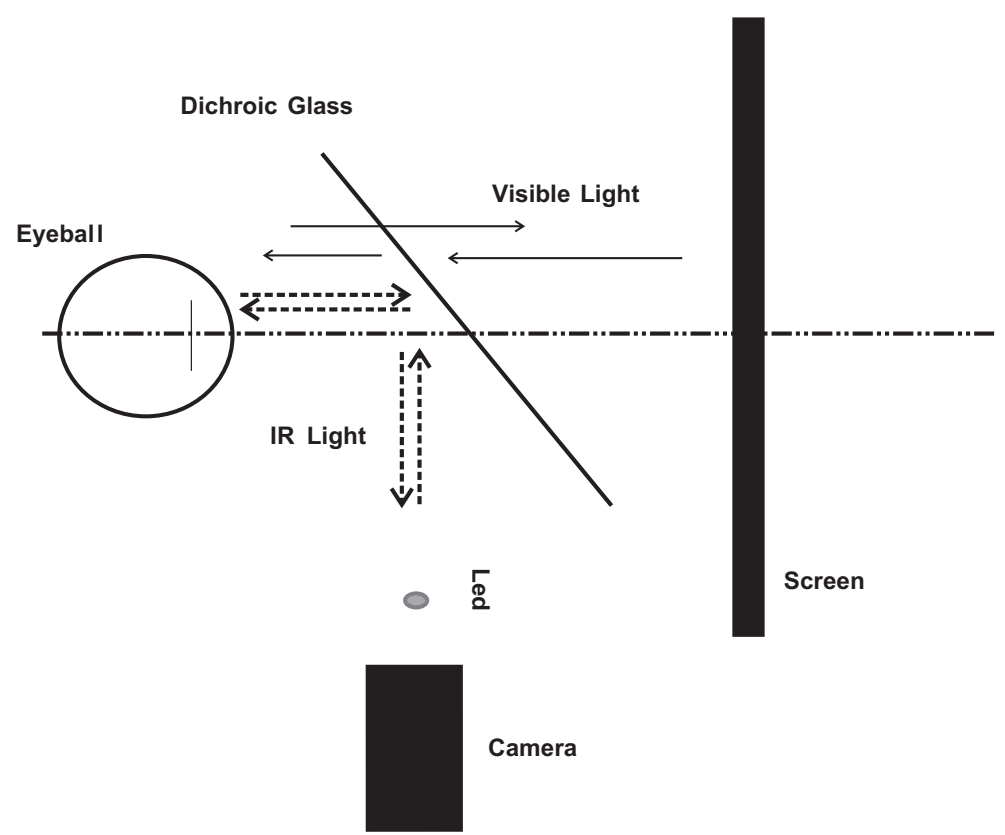

Fig. 5. Most simplistic configuration of the system using a dichroic glass.

1

3

5

reached by optical axis and $\left(p_{x v}, p_{y v}\right)$ point of the screen reached by visual axis (observed point).

The starting point is to carry out the modeling of the most simplistic configuration of the system elements, i.e. when the subject and the screen are perfectly aligned on the camera optical axis and consequently with the illumination source. Figure 5 shows a practical implementation of this arrangement using a dichroic glass. A dichroic glass reflects differently the light arriving to its surface depending on its wavelength. For the case of the figure it is clear that the used glass will be transparent to visible light going from the screen to user's eye, and will reflect the light emitted by the LED in such a way that it is perceived as it came from the center of the screen. In the same manner, the light reflected on the eye will be deviated to the camera by the glass. This is surely not the most practical setup but it is not the aim of the work to find the most robust system but to accomplish a deep mathematical review of the intrinsic nature of such systems. Due to its geometrical simplicity this configuration permits to extract interesting conclusions and allows to validate some specific results in an easier way.

\section{Building Up the Model: Optical and Visual Axes}

At this point of the work, we have gathered all the information needed to design the eye tracking system model we are looking for, i.e. $\left(p_{x v}, p_{y v}\right)$ and $\left(v d_{x}, v d_{y}\right)$ 


\section{$\mathbf{1}_{\text {st Reading }}$}

July 1, $2007 \quad$ 22:44 WSPC/115-IJPRAI $\quad$ SPI-J068 00569

Eye Tracking System Model Based on Physical Parameters

1 connection. The starting point, attending to the system arrangement explained in Sec. 3 , is the modeling of the optical axis $\left(p_{x o}, p_{y o}\right)$, that is directly related to the 3 pupil center in the image for the case shown in Fig. 5. Once the optical axis is modeled, the final objective is to accomplish the visual axis model, i.e. line of sight and consequently to derive the expressions for the gazed point $\left(p_{x v}, p_{y v}\right)$.

\subsection{Optical axis modeling}

$7 \quad$ The optical axis is assumed to be the eyeball symmetry axis and consequently it can be concluded that the center of the pupil will be located on this axis.

9 The pupil is assumed circular with radius $r$, and it is described in the eyeball coordinate system $\left(x_{1}, y_{1}, z_{1}\right)$ by

$$
\begin{aligned}
x_{1}^{2}+y_{1}^{2} & =r^{2} \\
z_{1} & =-t
\end{aligned}
$$

Projective geometry understanding and camera calibration parameters facilitate the transformation between the eyeball and image plane coordinate systems. ${ }^{2}$ Using homogeneous coordinates we have,

$$
\left(\begin{array}{c}
x \gamma \\
y \gamma \\
\gamma
\end{array}\right)=\left(\begin{array}{cccc}
\alpha f & 0 & u & 0 \\
0 & f & v & 0 \\
0 & 0 & 1 & 0
\end{array}\right)\left(\begin{array}{cc}
B_{\theta o} B_{\varphi o} & a \\
0 & 1
\end{array}\right)\left(\begin{array}{c}
x_{1} \\
y_{1} \\
z_{1} \\
1
\end{array}\right)
$$

where

$17-\left(x_{1}, y_{1}, z_{1}\right)$ : any point referred to the eyeball reference system

- $(x, y)$ : coordinates in the image plane

19

- $a:\left(a_{1}, a_{2}, a_{3}\right)$

21

- $B_{\theta o} B_{\varphi o}$ : eye rotation matrix

- $\gamma \neq 0$.

The eye rotation matrix will depend on the point to which the subject directs the optical axis. Assuming $a_{1}=a_{2}=0$ and anticlockwise rotations being positive we have,

$$
\begin{aligned}
& \sin \theta_{o}=\left(\frac{-\left(p o_{y 2}-p_{y o}\right)}{\sqrt{\left(a_{3}-p o_{z 2}\right)^{2}+\left(p o_{y 2}-p_{y o}\right)^{2}}}\right) \\
& \sin \varphi_{o}=\left(\frac{\left(p o_{x 2}-p_{x o}\right)}{\sqrt{\frac{\left(a_{3}-p o_{z 2}\right)^{2}}{\cos ^{2} \theta_{o}}+\left(p o_{x 2}-p_{x o}\right)^{2}}}\right)
\end{aligned}
$$

The obtained shape in the image plane is fully based on physical parameters of the eye tracking system. As explained in Sec. 2, two interesting features have to be extracted from the resulting image, i.e. glint position and center of pupil image. 


\section{$\mathbf{1}_{\text {st Reading }}$}

July 1, 2007 22:44 WSPC/115-IJPRAI SPI-J068 00569

10

A. Villanueva, R. Cabeza \&S S. Porta

1 The glint position is easily deduced considering the case in Fig. 5 that leads to the approximation $r_{x}=r_{y}=0$. On the other hand, the center of the projected shape

$3\left(m_{x}, m_{y}\right)$ is to be calculated. The projected shape of the pupil can be approximated to an ellipse ${ }^{1,15}$ in a wide range of gaze directions. This can be expressed through

5 system parameters employing the transformation matrix (5) and its center can be mathematically derived. ${ }^{2}$ However, the result is not totally satisfying, because it is extremely large and not a valid working expression due to its lack of simplicity.

The model thus obtained would not match one of the objectives imposed from the beginning because of its complexity. That is the reason to propose a new estimation that is described next.

In order to obtain the proposed model for the optical axis we approximate the center of the projected pupil to the projection of the center of the pupil, i.e. projection of the point $(0,0,-t)$ for the eyeball coordinate system $\left(x_{1}, y_{1}, z_{1}\right)$ in the image plane. One could think that both points, i.e. center of the projection and projection of the center are the same point, however due to projective relations this is not exactly true. In other words, the actual model takes the center $\left(m_{x}\right.$, $m_{y}$ ) in the image as the center of the resulting shape, whereas the approximated model considers the point of interest directly as the projection of the pupil center in the image plane, which is easily calculated employing the $3 \mathrm{D}-2 \mathrm{D}$ coordinates transformation matrix (5). For simplicity $u=v=0$ and $\alpha=1$.

$$
\begin{aligned}
& v d_{x}=-\frac{f\left(p o_{x 2}-p_{x o}\right) t}{a_{3}(\Delta-t)+p o_{z 2} t} \\
& v d_{y}=-\frac{f\left(p o_{y 2}-p_{y o}\right) t}{a_{3}(\Delta-t)+p o_{z 2} t}
\end{aligned}
$$

11 where,

$$
\Delta=\sqrt{\left(a_{3}-p o_{z 2}\right)^{2}+\left(p o_{x 2}-p_{x o}\right)^{2}+\left(p o_{y 2}-p_{y o}\right)^{2}}
$$

Anyway, the relation we are looking for is the expression relating the point of the screen with coordinates $\left(p_{x o}, p_{y o}\right)$ with the glint-pupil vector $\left(v d_{x}, v d_{y}\right)$; that is, the functional expressions

$$
\begin{aligned}
& p_{x o}=f\left(v d_{x}, v d_{y}\right) \\
& p_{y o}=g\left(v d_{x}, v d_{y}\right)
\end{aligned}
$$

From (8) and (9) we have,

$$
\begin{aligned}
& p_{x o}=p o_{x 2}+\frac{v d_{x}\left(a_{3}-p o_{z 2}\right)\left(f t^{2}+a_{3} \tau\right)}{a_{3}^{2}\left(v d_{x}^{2}+v d_{y}^{2}\right)-f^{2} t^{2}} \\
& p_{y o}=p o_{y 2}+\frac{v d_{y}\left(a_{3}-p o_{z 2}\right)\left(f t^{2}+a_{3} \tau\right)}{a_{3}^{2}\left(v d_{x}^{2}+v d_{y}^{2}\right)-f^{2} t^{2}}
\end{aligned}
$$




\section{$\mathbf{1}_{\text {st }}$ Reading}

July 1, $2007 \quad$ 22:44 WSPC/115-IJPRAI SPI-J068 00569

Eye Tracking System Model Based on Physical Parameters
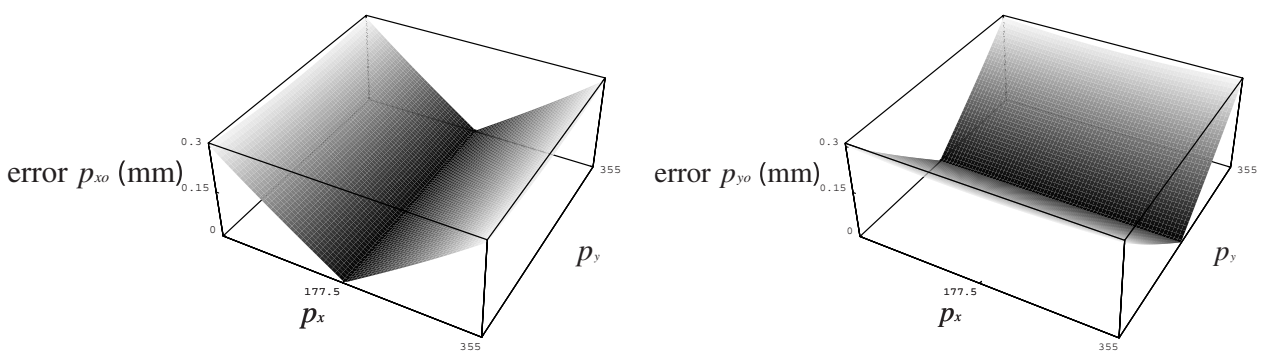

Fig. 6. Error distribution for $x$-coordinate and $y$-coordinate of optical axis over the screen.

where,

$$
\tau=\sqrt{f^{2} t^{2}-\left(v d_{x}^{2}+v d_{y}^{2}\right)\left(a_{3}^{2}-t^{2}\right)}
$$

These expressions represent a simple and suitable model. The symmetry exhibited by the model can be considered as a four-quadrant symmetry. In other words, for every point in the first quadrant of the screen three additional equivalent points can be defined in the remaining quadrants, all of them belonging to the same circumference. These points will have equal absolute values of glint-pupil vector, the signs varying depending on the quadrant. The arising error, due to the approximation of the point $\left(m_{x}, m_{y}\right)$ is symmetrical as well. For a standard working configuration $(355 \times 355 \mathrm{~mm}$ screen, subject's working distance from the camera $\sim 600 \mathrm{~mm}$, standard eyeball values) the error for both coordinates is graphically presented in Fig. 6. As deduced from the figures, the worst behavior takes place in the corners of the screen. In any case the obtained error value varies depending on the eyeball values, distance, screen and camera parameters.

The advantage of the model, besides its simplicity and symmetry, is that there are only two unknown parameters implied. Except $a_{3}\left(a_{1}=a_{2} \simeq 0\right)$ and $t$, that cannot be so easily calculated, the rest of involved variables can be accurately measured $\left(p o_{x 2}, p o_{y 2}, p o_{z 2}\right)$ or determined employing the camera calibration process $(f, u, v, \alpha)$. The indefinite parameters must be adjusted during the calibration session.

\subsection{Visual axis modeling}

From Sec. 3 we already know that visual axis modeling is not an obvious step; novel parameters have to be introduced in the eyeball model. As said before the aim of this work is to define a model that joins competence and simplicity, so it is desirable to reduce the number of parameters as much as possible. To this end the horizontal angular offset between optical and visual axes is just considered. ${ }^{15,18}$ The suggestion is to deduce the visual axis expression from the already obtained optical axis model. As the visual axis joins the fovea with a point (nodal point) close to the cornea center, the schematic model for the left eye will be modified as 


\section{$\mathbf{1}_{\text {st Reading }}$}

July 1, $2007 \quad$ 22:44 WSPC/115-IJPRAI $\quad$ SPI-J068 00569

12 A. Villanueva, R. Cabeza \& S. Porta

1 in Fig. 7, where two new adjustable parameters are added to the model:

- $\beta$ : horizontal angular offset between optical and visual axes.

3

- $w$ : distance between the eyeball center and the center of the corneal sphere.

We know from Sec. 2, the introduction of the visual axis does not only imply

5

7 modifications in the stationary or primary condition of the eyeball but also changes the relative position of both axes in tertiary positions due to the "false torsion" previously defined.

The "false torsion" is performed around the visual line and is a function of the angular rotations realized by this axis $\left(\theta_{v}, \varphi_{v}\right)$. It would be desirable to define this torsion related to the optical axis and as a function of the rotation accomplished by the eyeball coordinate system. In order to find the simplest model for the visual axis, the following approximation is proposed.

It is assumed $\alpha_{v}=\alpha_{o}, \alpha_{o}$ being the torsion performed around the $z_{1}$-axis of the eyeball coordinate system which, accordingly with the deduction made by Fry, ${ }^{7}$ can be fairly approximated as,

$$
\sin \alpha_{o}=\frac{\sin \theta_{o} \sin \varphi_{o}}{1+\cos \theta_{o} \cos \varphi_{o}}
$$

Expression (13) is the same as (3) except for the angles, now related to the optical axis. In addition, the negative sign disappeared due to the opposite directions of optical line and $z_{1}$-axis of the eyeball reference system.

Both optical and visual axes will always lie on the same plane. The issue is to infer the orientation of such plane referred to the camera coordinate system $\left(x_{2}, y_{2}, z_{2}\right)$. This task does not imply high difficulty. The only modification will be to change the eye rotation matrix from $B_{\theta_{o}} B_{\varphi_{o}}$ to $B_{\theta_{o}} B_{\varphi_{o}} B_{\alpha o}$ in (5). On the other hand, the visual axis would have to be expressed in the eyeball coordinate system $\left(x_{1}, y_{1}, z_{1}\right)$ as Fig. 7 shows. Any point of the visual axis will have coordinates $(-k \sin \beta, 0,-(w+k \cos \beta))$ with $k$ being real. Expressing the visual axis equation

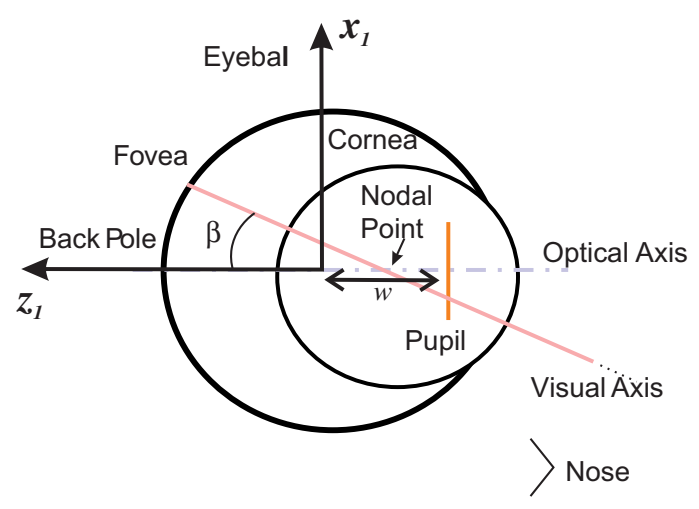

Fig. 7. Visual axis parameters. 


\section{$\mathbf{1}_{\text {st Reading }}$}

July 1, $2007 \quad$ 22:44 WSPC/115-IJPRAI $\quad$ SPI-J068 00569

as a function of optical axis is already feasible,

$$
\begin{aligned}
p_{x v}= & p o_{x 2}-\left(-\frac{\Delta_{4}\left(w+\lambda_{1} \cos \beta\right)}{\Delta_{6}}\right. \\
& \left.-\frac{\left(\Delta_{5}^{2}+\left(a_{3}-p o_{z 2}\right)\left(a_{3}-p o_{z 2}+\Delta_{6}\right)\right) \lambda_{1} \sin \beta}{\Delta_{6}\left(a_{3}-p o_{z 2}+\Delta_{6}\right)}\right) \\
p_{y v}= & p o_{y 2}-\left(-\frac{\Delta_{5}\left(w+\lambda_{1} \cos \beta\right)}{\Delta_{6}}-\frac{\Delta_{5} \Delta_{4} \lambda_{1} \sin \beta}{\Delta_{6}\left(a_{3}-p o_{z 2}+\Delta_{6}\right)}\right)
\end{aligned}
$$

where,

$$
\begin{aligned}
\Delta_{4} & =\left(p_{x o}-p o_{x 2}\right) \\
\Delta_{5} & =\left(p_{y o}-p o_{y 2}\right) \\
\Delta_{6} & =\sqrt{\left(a_{3}-p o_{z 2}\right)^{2}+\Delta_{4}^{2}+\Delta_{5}^{2}} \\
\lambda_{1} & =\frac{\left(a_{3}-p o_{z 2}\right)\left(-w+\Delta_{6}\right)}{\left(a_{3}-p o_{z 2}\right) \cos \beta-\Delta_{4} \sin \beta}
\end{aligned}
$$

The eye visual axis does not any longer present four-quadrant symmetry but preserves the regularity between the upper and lower halves of the screen, always for the case in Fig. 5. That is, the point $\left(p_{x v}, p_{y v}\right)$ will have an equivalence in $\left(p_{x v}\right.$, $\left.2 p o_{y 2}-p_{y v}\right)$.

Figures 8 shows the difference for $p_{x v}$ and $p_{y v}$ between the real and estimated values of the visual axis (for the left eye) without taking into account the optical axis approximation. The latter would have to be added to provide an overall estimation of the error for the built model (see Fig. 9). The visual axis approximations performed are different for $x$ - and $y$ - coordinates. The optical axis approach presents the highest contribution to the total error of the model for the horizontal coordinate. The nasal direction of the visual axis produces dissimilar behavior of the error between left and right sides of the screen, as expected. For the right eye the error would be easily deducible interchanging the figures symmetrically around the vertical axis of the screen.

Considering the optical axis as the line of sight $\left(p_{x o}, p_{y o}\right)=\left(p_{x v}, p_{y v}\right)$ leads to significant errors unaffordable for a gaze tracking mathematical model. From the
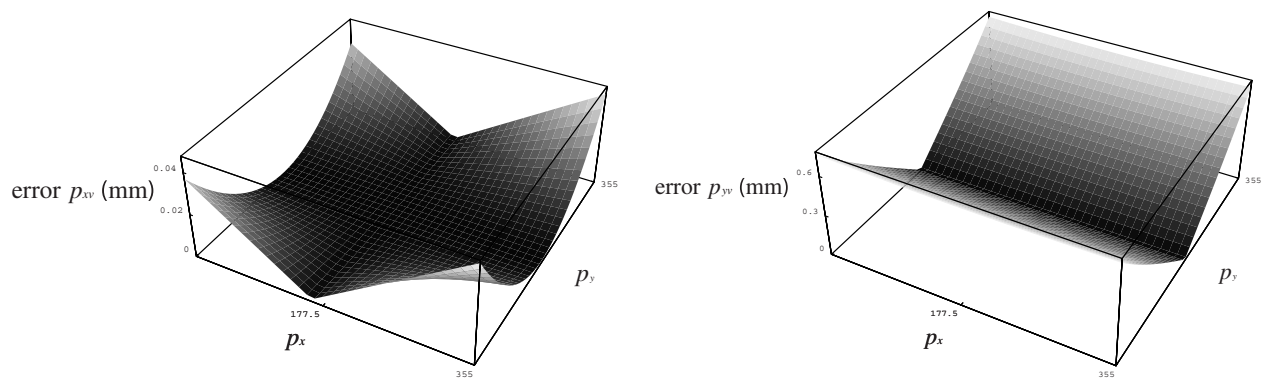

Fig. 8. Error distribution for $x$-coordinate and $y$-coordinate of visual axis over the screen. 

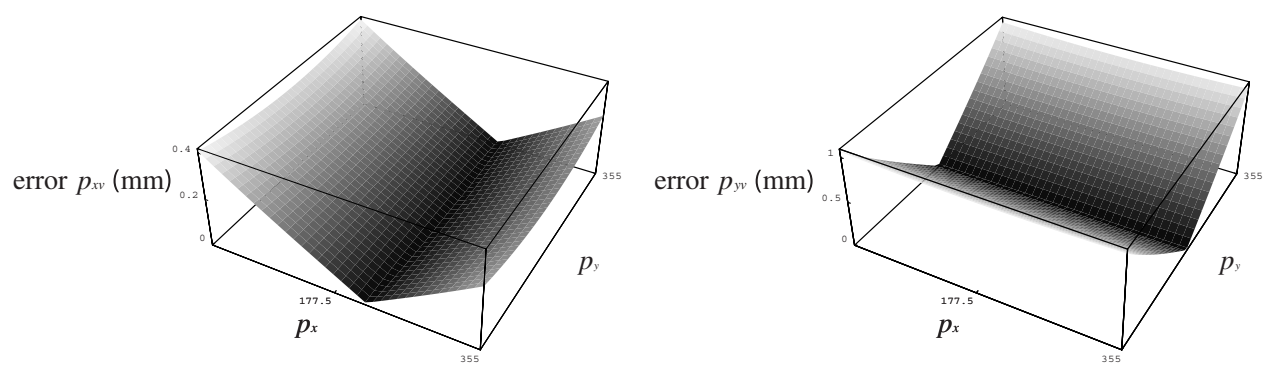

Fig. 9. Error distribution for $x$-coordinate and $y$-coordinate of visual axis over the screen considering optical axis approximation.

eyeball physiology we know that both axes present a standard angular offset of $5^{\circ}$. Consequently if the optical axis is considered as the line of sight an error of $\sim 5^{\circ}$ can be expected. Comparing this value with average accuracy that different eye tracking systems present nowadays (in the range of $0.3^{\circ}-1^{\circ}$ ) the possibility of considering the optical axis as the gaze line is rejected.

The number of parameters of the model influences considerably the calibration process that will be later exposed. As reducing the number of parameters is always desirable, the sensitivity of the error to the several adjustable parameters (i.e. $\left.t, \beta, a_{3}, w\right)$ is estimated. The method employed has been to vary these parameters in reasonable ranges and to compare the maximum error values arising on the screen with those obtained if the parameter were considered as a constant (considering its standard value). The variation ranges ${ }^{23}$ are $\left[3^{\circ}, 7^{\circ}\right]$ for $\beta,[7,15] \mathrm{mm}$ for $t$ and $[3,8] \mathrm{mm}$ for $w$. We have measured the arising differences between the visual axis coordinates employing parameter values within the specified ranges and resulting visual line values if the parameters were considered fixed for the same value of the glint-pupil vector. In addition it has been tested if the error can be compensated modifying the values of the rest of the parameters by means of calibration. For simplicity only the obtained conclusions will be emphasized, it is not affordable to take standard values for $\beta$ and $t$. The error increases considerably $\left(>1^{\circ}\right)$ and cannot be satisfactorily reduced to adjust the supplementary parameters by the calibration process. However, it has been demonstrated that the value of $w$ is negligible since the arisen error is easily compensated modifying $a_{3}$ about $1 \%$ as maximum.

So the expressions for visual axis will be slightly modified to obtain a simpler expression:

$$
\begin{aligned}
p_{x v}= & p o_{x 2}-\left(-\frac{\Delta_{4} \lambda_{2} \cos \beta}{\Delta_{6}}\right. \\
& \left.-\frac{\left(\Delta_{5}^{2}+\left(a_{3}-p o_{z 2}\right)\left(a_{3}-p o_{z 2}+\Delta_{6}\right)\right) \lambda_{2} \sin \beta}{\Delta_{6}\left(a_{3}-p o_{z 2}+\Delta_{6}\right)}\right) \\
p_{y v}= & p o_{y 2}-\left(-\frac{\Delta_{5} \lambda_{2} \cos \beta}{\Delta_{6}}-\frac{\Delta_{5} \Delta_{4} \lambda_{2} \sin \beta}{\Delta_{6}\left(a_{3}-p o_{z 2}+\Delta_{6}\right)}\right)
\end{aligned}
$$




\section{$\mathbf{1}_{\text {st }}$ Reading}

July 1, $2007 \quad$ 22:44 WSPC/115-IJPRAI $\quad$ SPI-J068 00569

Eye Tracking System Model Based on Physical Parameters

1

where,

$$
\lambda_{2}=\frac{\left(a_{3}-p o_{z 2}\right) \Delta_{6}}{\left(a_{3}-p o_{z 2}\right) \cos \beta-\Delta_{4} \sin \beta}
$$

The simplification achieved for the expressions is hardly noticeable but the reduction in the number of parameters and its benefit for the calibration fully justifies the omission of this variable. The last step will be to replace $\left(p_{x o}, p_{y o}\right)$ in the previous expressions by the model for the optical axis (11)-(12) to reach our objective, i.e. to find the relation between the glint-pupil vector $\left(v d_{x}, v d_{y}\right)$ and the point on the screen the subject is looking at $\left(p_{x v}, p_{y v}\right)$. Once the system is modeled alternative calibration methods should be searched.

\section{Calibration}

As previously explained, in a calibration process the subject is asked to look at some marks or points on the screen. The normally used patterns consist several points disposed in a $3 \times 3$ or $4 \times 4$ grid.

This process can sometimes be boring and tiresome for users. As the reduction of calibration processes is one of the objectives of eye tracking systems developers, it would be desirable to have a model able to allow an easy calibration procedure. Once the subject has looked sequentially to the marks, data are collected and an adjustable model is adapted. In case of generic mathematical models the coefficients of the expressions have to be deduced to fit the calibration points with the lowest possible error.

Given the proposed model and a calibration resulting data set of type $\left(v d_{x i}\right.$, $\left.v d_{y i}, p_{x v i}\right),\left(v d_{x i}, v d_{y i}, p_{y v i}\right)$, the regression process adjusts the parameters of the model (that will be different for each coordinate) $a_{3}, t$ and $\beta$ to fit the equations for $p_{x v}$ and $p_{y v}$ to the given session and the certain subject working with the system. Mathematically the number of adjustable parameters of the selected model will determine the number of points to calibrate in order to determine parameter values. Consequently three points of calibration will be enough to adjust the proposed model.

Working with a nongeneric model (like the one proposed) presents several advantages compared to general-purpose expressions, linear or quadratic. First, a model completely based on real parameters allows to know the values of a considerable number of variables before calibration, so there are less parameters to be adjusted. Second, the symmetries presented by the model are already known, and this implies to identify areas of the screen that must have similar behavior. Another consequence of such a model is to recognize directly from its expression those points that surely will not provide any information in a fitting process. For example, if the optical axis is going to be adjusted, it has no sense to select the point $\left(p_{x o}=p o_{x 2}, p_{y o}=p o_{y 2}\right)$ as calibration point. From (11)-(12) we already know that $v d_{x}=0 \Leftrightarrow p_{x}=p o_{x 2}$ and $v d_{y}=0 \Leftrightarrow p_{y}=p o_{y 2}$ so there is no parameter to be fitted. In the same manner, taking model symmetrical points do not provide the best contribution for the 


\section{$\mathbf{1}_{\text {st }}$ Reading}

July 1, $2007 \quad$ 22:44 WSPC/115-IJPRAI $\quad$ SPI-J068 00569

1 regression process, because they will represent a unique input. These conclusions were confirmed in the work by Villanueva. ${ }^{22}$

In the end, if the optical axis model has to be calibrated, two points are enough because just two parameters $\left(a_{3}, t\right)$ need to be fitted, and they can be located in the same quadrant. On the other hand, three calibration marks, i.e. three points, are needed to adjust the pursued visual axis model parameters $\left(a_{3}, t, \beta\right)$ for both coordinates. For this case the symmetry imposes the possibility of calibrating the system using just the upper $\left(p_{y v} \leq p_{y_{2}}\right)$ or lower $\left(p_{y v} \geq p o_{y 2}\right)$ half of the screen.

A simulation has been made in order to measure the performance of the model in a theoretical stage. The figures of merit considered to measure the competence of the adjusted model, after calibration, are two: the arising maximum and average errors. Once the model is adjusted, the estimated values for the visual axes of a uniformly distributed number of points are calculated and compared to the real ones in order to obtain both errors. These errors are dependent on the particular points selected for regression and if a suitable calibration group of markers is chosen the resulting model after regression presents an accurate tracking capability. Conjugate gradient and BFGS quasi-Newton methods have been employed to find such most adequate calibration points for a standard set of values with a screen size of $355 \times 355 \mathrm{~mm}$ and a working distance of $600 \mathrm{~mm}$. The outcoming results showed that heuristically the maximum error for $p_{x v}$ remains around $0.0147 \mathrm{~mm}$ with an average difference of $0.0054 \mathrm{~mm}$. For $p_{y v}$, the errors are lower: $0.0108 \mathrm{~mm}$ and $0.0038 \mathrm{~mm}$ as maximum and average values respectively $\left(<0.01^{\circ}\right)$.

Evidently the exact location of the points will depend on the configuration data (screen dimensions, camera parameters, subject) for the working session. However some guidelines can be extracted from the analysis that lead to a proper selection of calibration points.

First, and taking into account the symmetry of the model, it is not necessary to consider points in both upper and lower parts of the screen; calibration over one of the screen halves fits the model at point of the monitor.

Points in the corners have to be taken into account, as they present the most noticeable errors. Considering a point for regression improves the accuracy of the model around that certain marker; so maximum errors will become lower, reducing as well the weight in the overall average inaccuracy, if a point in one of the corners is taken for regression.

Due to the lack of symmetry between the models $p_{x v}$ and $p_{y v}$ the best group of calibration points for both coordinates do not coincide. Actually the most suitable regression markers can be different for the $x$ - and $y$-coordinates of the visual axis, although an average calibration with a competent behavior for both coordinates is always possible to be found. Depending on the eye to be calibrated the location of the best calibration points will vary. For the left eye it seems convenient to take two points in the left part of the screen and one on the right part, due to different error values arising in both parts of the screen and produced by the nasal orientation of 


\section{$\mathbf{1}_{\text {st Reading }}$}

July 1, $2007 \quad$ 22:44 WSPC/115-IJPRAI $\quad$ SPI-J068 00569

Eye Tracking System Model Based on Physical Parameters

1

3

the visual axis with respect to the optical line of the eye. The same premise leads to make the opposite selection for the right eye.

The experimental validation carried out in next section corroborates these conclusions. It will be shown that only three points are necessary to fit the model and to achieve a performance comparable to a generic mathematical model that needs nine calibration points, thus reducing considerably time and efforts for the subject calibrating the system.

\section{Experimental Validation}

The system employed for the experimental validation uses a Hamamatsu C5999 camera and a Matrox Meteor acquisition card with a resolution of $640 \times 480$ (RS170). The system works at $60 \mathrm{~Hz}$. The LED used for lighting has a spectrum centered at $850 \mathrm{~nm}$. The whole system is controlled by a dual processor Pentium at $1.7 \mathrm{GHz}$ with $256 \mathrm{MB}$ of RAM. The development environment has been Visual $\mathrm{C}++$ under Windows 2000. It is not the aim of this paper to give detailed information about the image processing methods, it can be found in the paper by Goñi ${ }^{8}$ and Villanueva. ${ }^{21}$

Three subjects located about $\sim 600 \mathrm{~mm}$ from the camera observed $15+18$ points on a screen of $355 \times 355 \mathrm{~mm}$. The performance of the designed model (model2) is compared to that from a competent generic mathematical model (model1).

Calibration for Model 1: Generic Model

Such generic model is defined as,

$$
\begin{aligned}
p_{x v}= & a_{x} v d_{x}^{2} v d_{y}^{2}+b_{x} v d_{x}^{2} v d_{y}+c_{x} v d_{x}^{2}+d_{x} v d_{y}^{2}+ \\
& +e_{x} v d_{x} v d_{y}^{2}+f_{x} v d_{x}+g_{x} v d_{y}+h_{x} v d_{x} v d_{y}+k_{x} \\
p_{y v}= & a_{y} v d_{x}^{2} v d_{y}^{2}+b_{y} v d_{x}^{2} v d_{y}+c_{y} v d_{x}^{2}+d_{y} v d_{y}^{2}+ \\
& +e_{y} v d_{x} v d_{y}^{2}+f_{y} v d_{x}+g_{y} v d_{y}+h_{y} v d_{x} v d_{y}+k_{y}
\end{aligned}
$$

Figure 10 shows the calibration points (on the left part). For the generic model, the subject is forced to look at 9 of the 15 points shown (points 1-9). The generic expressions are adjusted through a regression process employing the information $\left(v d_{x i}, v d_{y i}, p_{x v i}\right),\left(v d_{x i}, v d_{y i}, p_{y v i}\right)$ obtained from $3 \times 3$ calibration grid. Then the adjusted model tracking capability is measured calculating the real and estimated values for the same 15 points plus 18 more test points, that are shown together on the right in Fig. 10. These 18 points have been arbitrarily selected to assure that the model behavior has nothing to do with any particular pattern or points distribution. The error is calculated over the $15+18$ points.

Calibration for Model 2: Proposed model

This model is expressed by Eqs. (16) and (17) obtained in Sec. 5 where $\left(p_{x o}, p_{y o}\right)$ are replaced by expressions (11) and (12).

For the proposed model the best list of three candidates is to be determined among the 15 proposed points (Fig. 10 (left), points 1-15). For each possible group 


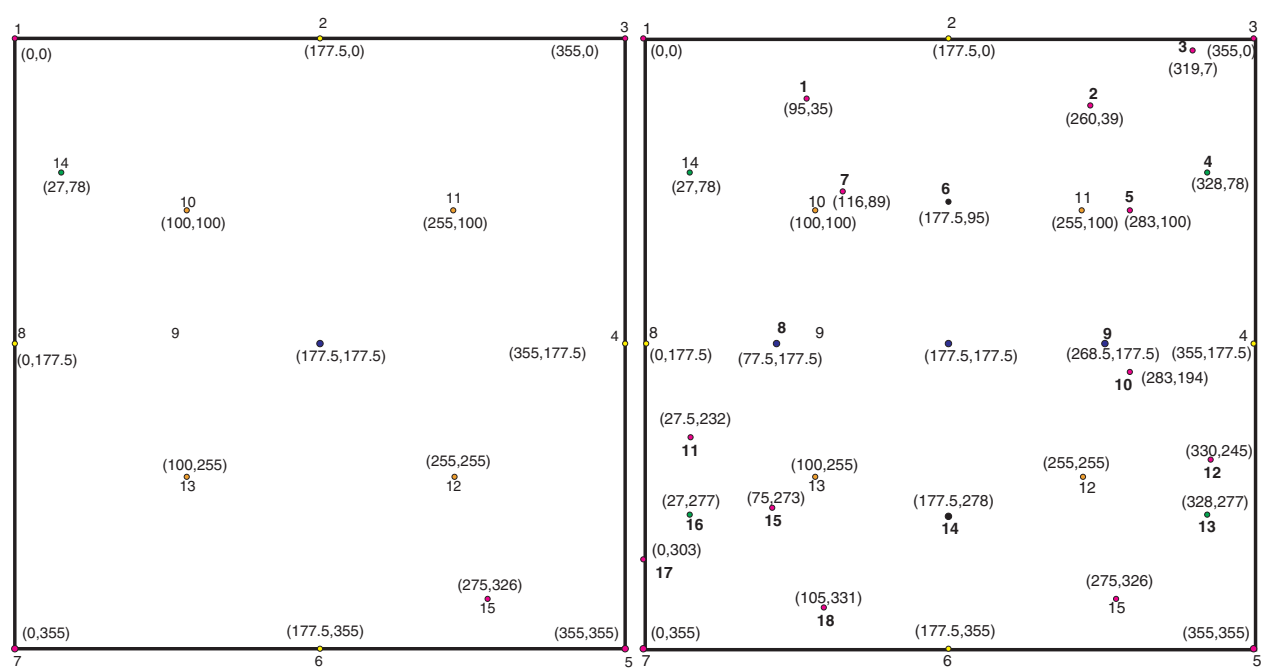

Fig. 10. Calibration and test points. (Left) Calibration points; points 1-9 are used for the generic model, sets of three points among the fifteen are employed to adjust the proposed model. (Right) Both models are tested over the $15+18$ points shown.

of three points a calibration can be made. Once the model is adjusted its performance has to be tested for the remaining $15+18$ points in the same way as for the generic model.

Tables 1 (for the $p_{x v}$ coordinate) and 2 (for the $p_{y v}$ coordinate) aim to show the maximum and average errors resulting from the regressions for the generic model (model1) calibrated with 9 points and for the proposed one (model2) calibrated with 3 points. For the latter, some of the best calibration groups are given. The values shown are obtained after averaging the results from the three subjects. In the first column the model selected is shown. For the proposed model the set of points considered for calibration is given. In the second column the obtained maximum and average errors are given.

When dealing with real subjects and real systems an indetermination in the resulting values is always to be assumed. This inaccuracy and the tolerance of the

Table 1. Maximum and average errors comparison for $p_{x v}$.

\begin{tabular}{lcc}
\hline & $p_{x v}$ & \\
& $\left(\right.$ max err. $p_{x v}$, avg err. $\left.p_{x v}\right)(\mathrm{mm})$ & Avg err.over 18 test points $(\mathrm{mm})$ \\
\hline Generic model $(3 \times 3)$ & $(5.898,1.460)$ & 2.337 \\
Proposed model & & \\
$(1,4,8)$ & $(5.079,1.965)$ & 2.391 \\
$(1,4,14)$ & $(5.784,1.948)$ & 2.371 \\
$(4,5,15)$ & $(5.351,1.901)$ & 2.337 \\
$(7,4,8)$ & $(4.651,2.005)$ & 2.344 \\
$(7,4,14)$ & $(4.918,1.885)$ & 2.288 \\
\hline
\end{tabular}




\section{$\mathbf{1}_{\text {st }}$ Reading}

July 1, $2007 \quad$ 22:44 WSPC/115-IJPRAI $\quad$ SPI-J068 00569

Eye Tracking System Model Based on Physical Parameters

Table 2. Maximum and average errors comparison for $p_{y v}$.

\begin{tabular}{|c|c|c|}
\hline & $\begin{array}{c}p_{y v} \\
\left(\max \text { err. } p_{y v}, \text { avg err. } p_{y v}\right)(\mathrm{mm})\end{array}$ & Avg err.over 18 test points $(\mathrm{mm})$ \\
\hline Generic model $(3 \times 3)$ & $(7.456,2.193)$ & 2.894 \\
\hline \multicolumn{3}{|l|}{ Proposed model } \\
\hline$(3,7,15)$ & $(6.303,2.940)$ & 2.268 \\
\hline$(1,3,15)$ & $(6.013,2.508)$ & 2.217 \\
\hline$(1,5,15)$ & $(5.464,1.931)$ & 1.774 \\
\hline$(1,10,11)$ & $(6.271,2.346)$ & 2.413 \\
\hline$(1,11,13)$ & $(7.102,2.309)$ & 2.663 \\
\hline
\end{tabular}

1

configuration data as well as misalignments among eye tracking system elements reflects in higher arising errors for the model compared to the theoretical values. However, the desired performance is fully achieved and moreover the sufficiency of just three points renders a more competent and practical model.

From these tables it is clear that the proposed model gets comparable maximum errors. The average error is quite misleading, since improvement seems worse than that achieved for the maximum error. However the points considered for the average error fully determine the obtained value. Higher average error for the generic model is expected if more possible noncalibrated points on the screen are considered. In other words, the ratio (number of test points)/(number of calibration points) for the proposed model is $\sim 3$ times the ratio for the generic one, which leads to a different amount of calibrated points among the set of test points. The average errors shown in the table are calculated considering $15+18$ test points. From these points 9 are used to calibrate the generic model and 3 for the proposed one. After the fitting process low errors are expected at the points used for the calibration. A more realistic value would be obtained if for both cases noncalibrated points were used to test the average error. To this end an additional analysis is made; the average error is calculated just over 18 noncalibrated test points (in bold in Fig. 10 (right)) and the results are shown in the third column of the table. As expected the average behavior of the proposed model is improved compared to the errors shown in the second column after this analysis.

It is possible that the limited set of calibration points selected does not contain the best regression marks for the configuration data of the sessions carried out. So surely there is a set of three points in the screen that could have produced a better performance for the given subject and system configuration. Anyway it always depends on the session and system setup; consequently their positions can be hardly specified previously. The objective is to specify a set of points that will assure a competent behavior for both coordinates in a standard working session. Having the proper calibration conditions the guidelines given in the previous section, i.e one point in the corner (highest error) and two points in the left part of the screen (for left eye), will necessarily lead to an efficient and highquality model specification. Example of calibrations with a good performance for both coordinates simultaneously is the trio $(1,14,3)$ with the following errors for 


\section{$\mathbf{1}_{\text {st }}$ Reading}

July 1, $2007 \quad$ 22:44 WSPC/115-IJPRAI $\quad$ SPI-J068 00569

20

A. Villanueva, R. Cabeza \&S S. Porta

$1 \quad p_{x v}$ and $p_{y v},(5.308,2.041),(7.187,2.373)$; and $(1,10,15)$ with errors $(5.380,1.902)$, $(6.557,1.910)$ for the same coordinates of the visual axis.

When dealing with gaze tracking system it is more appropriate to give the error in visual degrees. Considering the average values of the errors, we have that the generic model presents an error of $0.35^{\circ}$, and the proposed one shows a value of $0.25-0.29^{\circ}$. Both values are in the acceptable ranges, i.e. $<1^{\circ}$.

It is well known how sensitive eye tracking systems are to the working distance. When the distance between the camera and the subject varies image processing problems can appear and modifying the calibration distance can affect the tracking model and increase the gaze determination error. A simulation has been carried out for a standard case $(\sim 500 \mathrm{~mm}$, standard eyeball $)$ to measure this sensitivity in a more systematic way. Once both models, i.e. the proposed model and the generics one are calibrated, the working distance is changed to compare their performances. Both models have shown a similar behavior. The distance variation is critical for both models: error keeps below $1^{\circ}$ if distance variation does not exceed $4 \%$. For this standard case the proposed model is superior to the generic one, i.e. presents lower errors, as the working distance decreases. On the contrary the generic one exhibits a better behavior when the working distance increases. However the arisen differences are not significative to conclude the superiority of any of the models regarding this problem.

\section{Conclusions and Future Work}

Mathematical modeling is one of the less revised aspects of eye tracking systems, maybe because generic expressions commonly employed do present an acceptable performance in standard working conditions. However, a deeper knowledge about the geometry and symmetry of the problem may lead to design more robust models. It is of great interest having available expressions relating the elements of the eye tracking system in order to measure the sensitivity to specific parameters of the tracker and to help explain abnormal behaviors under alternative working conditions.

The system used in this work employs the well-known bright-pupil technique to track the subject's gaze. The objective of the work has been to model the tracker using geometric and eyeball kinematics knowledge together in order to find the most realistic, simple and competent tracking model. Optical axis modeling has been the first step of the work, to finally achieve the outcoming visual axis expression derived through a deep analysis of eyeball kinematics. The estimations for both optical and visual axes have been based on geometrical approaches that have resulted in expressions that faithfully preserve the symmetry of the real system. Simplicity has been an additional attribute in the searching for the best possibilities, finding a trade-off between competence and complexity.

The benefits of the proposal are clear: it presents a review and a parametrical description of the elements forming the eye tracking system that constitutes a 


\section{$\mathbf{1}_{\text {st }}$ Reading}

July 1, $2007 \quad$ 22:44 WSPC/115-IJPRAI $\quad$ SPI-J068 00569

Eye Tracking System Model Based on Physical Parameters

mathematical basis that can lead to new results and better system behavior control. It is fully based on identifiable parameters related to physical elements of the system, it preserves the symmetry between the upper and lower parts of the screen and employs just three unknowns, which renders the main advantage of the model that is the simple three-point calibration. It has been demonstrated that the model presents high-quality performance after a three-point regression, more than comparable with the behavior of generic mathematical expressions using nine points for fitting purposes.

Avoiding the bad calibration points indicated, a three-point calibration provides an acceptable behavior for the proposed model. The performance of the model can vary depending on the set of three points selected. The best set of points will change as the system arrangement or the subject varies. Although some guidelines have been given in order to find out adequate calibration points, the proper way to determine the best calibration marks for a given system is actually an interesting research line for the future.

The indetermination of the experimental data, possible misalignments among the elements (camera, eye, screen and illumination), as well as tolerances in the values of physical magnitudes of the elements lead to higher errors than the ones calculated heuristically. Although an acceptable model behavior has been achieved, we expect to improve the excellence of the tracking system modifying the model in order to detect possible misalignments, to manage the indetermination related to the captured data and making a more accurate analysis of the glint position in the image. Model improvement as well as new system configurations development appear as promising objectives in the future. In addition, including more image features such as more glints or pupil shape as system features is surely going to render alternative gaze tracking models with broad possibilities.

\section{References}

1. D. Beymer and M. Flickner, Eye gaze tracking using an active stereo head, Proc. IEEE Conf. Computer Vision and Pattern Recognition (Wisconsin, 2003) 2, pp. 451-458.

2. W. Boehm and H. Prautzsch, Geometric Concepts for Geometric Design (AK Peters Ltd., Wellesley, MA, 1994).

3. RHS. Carpenter, Movements of the Eyes (Pion Limited, London, 1988).

4. T. N. Cornsweet and H. D. Crane, Accurate two-dimensional eye tracker using first and fourth Purkinje images, J. Opt. Soc. Amer. 63 (1973) 921-928.

5. Y. Ebisawa and S. Satoh, Effectiveness of pupil area detection technique using two light sources and image difference method, Proc. 15th Ann. Int. Conf. IEEE Engineering in Medicine and Biology Society (San Diego 1993), pp. 1268-1269.

6. L. Ferman, H. Collewijn and A. V. Van Den Berg, A direct test of Listing's LawI. Human ocular torsion measured in static tertiary positions, Vis. Res. 27 (1987) 929-938.

7. G. A. Fry, C. L. Treleaven, R. Walsh, E. L. Higgins and C. A. Radde, Definition and measurement of torsion, Amer. J. Opt. Arch. Amer. Acad. Opt., Philadelphia, 24 (1947) 329-334. 


\section{$\mathbf{1}_{\text {st Reading }}$}

July 1, $2007 \quad 22: 44 \quad$ WSPC/115-IJPRAI $\quad$ SPI-J068 00569

8. S. Goñi, J. Echeto, A. Villanueva and R. Cabeza, Robust algorithm for pupil-glint vector detection in a video-oculography eyetracking system, Proc. Int. Conf. Pattern Recognition (Cambridge, 2004), pp. 941-944.

9. A. Haro, M. Flickner and I. Essa, Detecting and tracking eyes by using their physiological properties dynamics and appearance, Proc. IEEE CVPR (South Carolina, 2000), pp. 163-168.

10. R. J. K. Jacob, The use of eye movements in human-computer interaction techniques: What you look at is what you get, ACM Trans. Inform. Syst. 9(3) (1991) 152-169.

11. J. Merchant, R. Morrissette and J. L. Porterfield, Measurement of eye direction allowing subject motion over one cubic foot of space, IEEE Trans. Biomed. Engin. 21(4) (1974) 309-317.

12. C. H. Morimoto, D. Koons, A. Amir and M. Flickner, Pupil detection and tracking using multiple light sources, Image Vis. Comput. 18(4) (2000) 331-336.

13. O. H. Mowrer, R. C. Ruch and N. E. Miller, The corneo-retinal potential difference as the basis of the galvanometric method of recording eye movements, Amer. J. Physiol. 114 (1936) 423.

14. K. Nakayama and R. Balliet, Listing's Law, eye position sense and perception of the vertical, Vis. Res. 17 (1977) 453-457.

15. T. Ohno and N. Mukawa, A free head, simple calibration, gaze tracking system that enables gaze-based interaction, Proc. Eye Tracking Research 83 Applications Symp., (San Antonio, TX, 2004), pp. 115-122.

16. D. Ott, F. Gehle and R. Eckmiller, Video-oculographic measurement of 3-dimensional eye rotations, J. Neurosci. Meth. 35 (1990) 229-234.

17. R. B. Rabbetts, Bennett \& Rabbetts clinical visual optics, 3rd Edition (ButterworthHeinemann, Elsevier Ltd., Edinburgh, 1998).

18. S. Shih and J. Liu, A novel approach to 3-D gaze tracking using stereo cameras, IEEE Trans. Syst. Man Cybern. Part-B 34 (2004) 234-245.

19. A. Tomono, M. Iida and Y. Kobayashi, A TV camera system which extracts feature points for non-contact eye movements detection, Proc. SPIE Optics, Illumination and Image Sensing for Machine Vision IV, 1194 (1990), pp. 2-12.

20. E. Trucco and A. Verri, Introductory Techniques for 3-D Computer Vision (Prentice Hall, New Jersey, 1998).

21. A. Villanueva, M. Cuellar, R. Cabeza and S. Porta, Real time video-oculography system, Proc. 11th European Conf. Eye Movements (Turku, Finland, 2001), pp. 51.

22. A. Villanueva, R. Cabeza and S. Porta, Eye tracking system model with easy calibration, Proc. Eye Tracking Research \& Applications Symposium (San Antonio, TX, 2004), pp. 55.

23. A. L. Yarbus, Eye Movements and Vision (Plenum Press, NY, 1967).

24. L. R. Young and D. Sheena, Survey of eye movement recording methods, Methods \& Designs, Behav. Res. Meth. Instrument. 7(5) (1975) 397-427. 